\title{
Virological and Immunological Outcomes in Rhesus Monkeys after Exposure to Dengue Virus-Infected Aedes aegypti Mosquitoes
}

\author{
Lauren B. Carrington, ${ }^{1,2 \star}$ Alongkot Ponlawat, ${ }^{3}$ Chanyapat Nitatsukprasert, ${ }^{3}$ Patcharee Khongtak, ${ }^{3}$ Piyanate Sunyakumthorn, ${ }^{3}$ \\ Christine A. Ege, ${ }^{3}$ Rawiwan Im-erbsin, ${ }^{3}$ Kesara Chumpolkulwong, ${ }^{3}$ Butsaya Thaisomboonsuk, ${ }^{3}$ Chonticha Klungthong, ${ }^{3}$ \\ In-Kyu Yoon, ${ }^{3}$ Damon Ellison, ${ }^{3}$ Louis Macareo, ${ }^{3}$ and Cameron P. Simmons ${ }^{1,2,4}$ \\ ${ }^{1}$ Oxford University Clinical Research Unit (OUCRU), Wellcome Trust Asia-Africa Programme, Ho Chi Minh City, Vietnam; ${ }^{2}$ Nuffield Department of \\ Medicine, University of Oxford, Oxford, United Kingdom; ${ }^{3}$ Armed Forces Research Institute of Medical Sciences (AFRIMS), Bangkok, Thailand; \\ ${ }^{4}$ Institute for Vector-Borne Diseases, Monash University, Melbourne, Australia
}

\begin{abstract}
This study describes the natural history of dengue virus (DENV) infection in rhesus monkeys exposed to the bites of DENV-infected Aedes aegypti mosquitoes. Dengue virus-infected mosquitoes were generated by either intrathoracic inoculation or by oral feeding on viremic blood meals. Each of the six rhesus monkeys that were fed upon by intrathoracically infected mosquitoes developed non-structural protein 1 (NS1) antigenemia and an IgM response; viremia was detected in 4/6 individuals. No virological or immunological evidence of DENV infection was detected in the three monkeys exposed to mosquitoes that had been orally infected with DENV. These results demonstrate the utility of mosquito-borne challenge of rhesus monkeys with DENV.
\end{abstract}

\section{INTRODUCTION}

Dengue is the most widespread arthropod-borne viral disease in the world. The causative agent, dengue virus (DENV), is transmitted in a human-mosquito-human transmission cycle, primarily involving Aedes aegypti mosquitoes. Intervention strategies aimed at inhibiting the transmission cycle of DENV between humans and mosquitoes are at various stages of testing. One of these strategies involves the use of Wolbachia to reduce the vector competence of Ae. aegypti populations for medically important arboviruses. ${ }^{1-3}$ Conventionally, researchers assess mosquito vector competence in an in vitro transmission (IVT) assay. The IVT assay involves the restraint and subsequent collection of saliva from individual mosquitoes ${ }^{4}$ which is then tested for the presence of virus, using either direct or indirect detection methods. ${ }^{5-10}$

It is unknown how well the laboratory-based IVT assay correlates with the actual transmission potential, that is, the probability of virus transmission during the bite of an infected mosquito on a susceptible host. Nonhuman primates (NHPs) offer a model animal system that can be used to validate and calibrate IVT assays. Rhesus macaques (Macaca mulatta) are susceptible to DENV infection and develop a viremia and immune response but do not develop signs or symptoms of infection. ${ }^{11-16}$ Recent work on Zika virus (ZIKV) infection in macaques demonstrates that the mode of virus delivery can alter the course of infection. Relative to infection via subcutaneous inoculation, delivery of virus through the bite of a ZIKV-infected Ae. aegypti prolonged the peak viremia in the animals and increased the virus sequence heterogeneity in the resulting virus population. ${ }^{17}$ Comparable studies of viral kinetics in response to DENV delivered by mosquito bites have not been performed to date. In the only identified study in which NHPs were infected via the bite of infectious mosquitoes, ${ }^{16}$ the infection outcome was measured serologically 28 days after exposure; viremia and NS1 antigenemia profiles were not measured.

This study was motivated by the desire to establish a reliable mosquito-to-NHP infection model that would subsequently

* Address correspondence to Lauren B. Carrington, Oxford University Clinical Research Unit (OUCRU), 764 Vo Van Kiet, District 5, Ho Chi Minh City, 70000, Vietnam. E-mail: Ibcarrington@gmail.com allow us to measure the efficacy of Wolbachia strains to block transmission. In doing so, we aimed at calibrating the laboratory-based IVT assay to result in the NHP infection model.

\section{METHODS}

Study outline. Three independent experiments were conducted. Each experiment involved three rhesus macaques, each directly fed upon by three DENV-infected Ae. aegypti. Some variations in the methodological approach between the first and subsequent experiments were necessary, and these are described in the following appropriate sections (Mosquito rearing and origin, and Viral infection of mosquitoes [day -14]).

A schematic diagram of the study design is depicted in Figure 1. In brief, mosquitoes were first infected with virus on day -14 , either by oral challenge using viremic blood from an acute dengue patient (as in Experiment 1) or by direct inoculation of virus into the mosquito (Experiments 2 and 3 ). Two weeks later, DENV-infected mosquitoes were allowed to feed on NHPs, representing day 0 . For the next 15 days, NHPs were monitored with intermittent blood sampling at 3-day intervals, coupled with a final day 28 time point to resolve serological status. Every blood sample collected was tested by DENV polymerase chain reaction (PCR), NS1 ELISA, and IgM/ IgG ELISA tests. To compare outcomes in the macaque infection model and the IVT assay, the day following the NHPmosquito exposure (day 1), we tested saliva and salivary glands from each of the nine mosquitoes that fed upon NHPs using our standard IVT protocol. ${ }^{5,6,10}$

Ethical considerations. This study was reviewed and approved by the IACUC and Biosafety Review Committee at the Armed Forces Research Institute for Medical Science (AFRIMS), an AAALAC International-accredited facility (protocol number 14-04). All NHP-related research were conducted in accordance with Thai laws; the Animal Welfare Act; all applicable U.S. Department of Agriculture, Office of Laboratory Animal Welfare, U.S. Department of Defense guidelines; and EU standards for NHP research, including housing for NHPs in European ETS123 standard cages. Nonhuman primates were phlebotomized voluntarily during the course of the study (days 3, 6, 9, 12, and 15); only the first and final blood 
In vitro virus transmission by mosquitoes

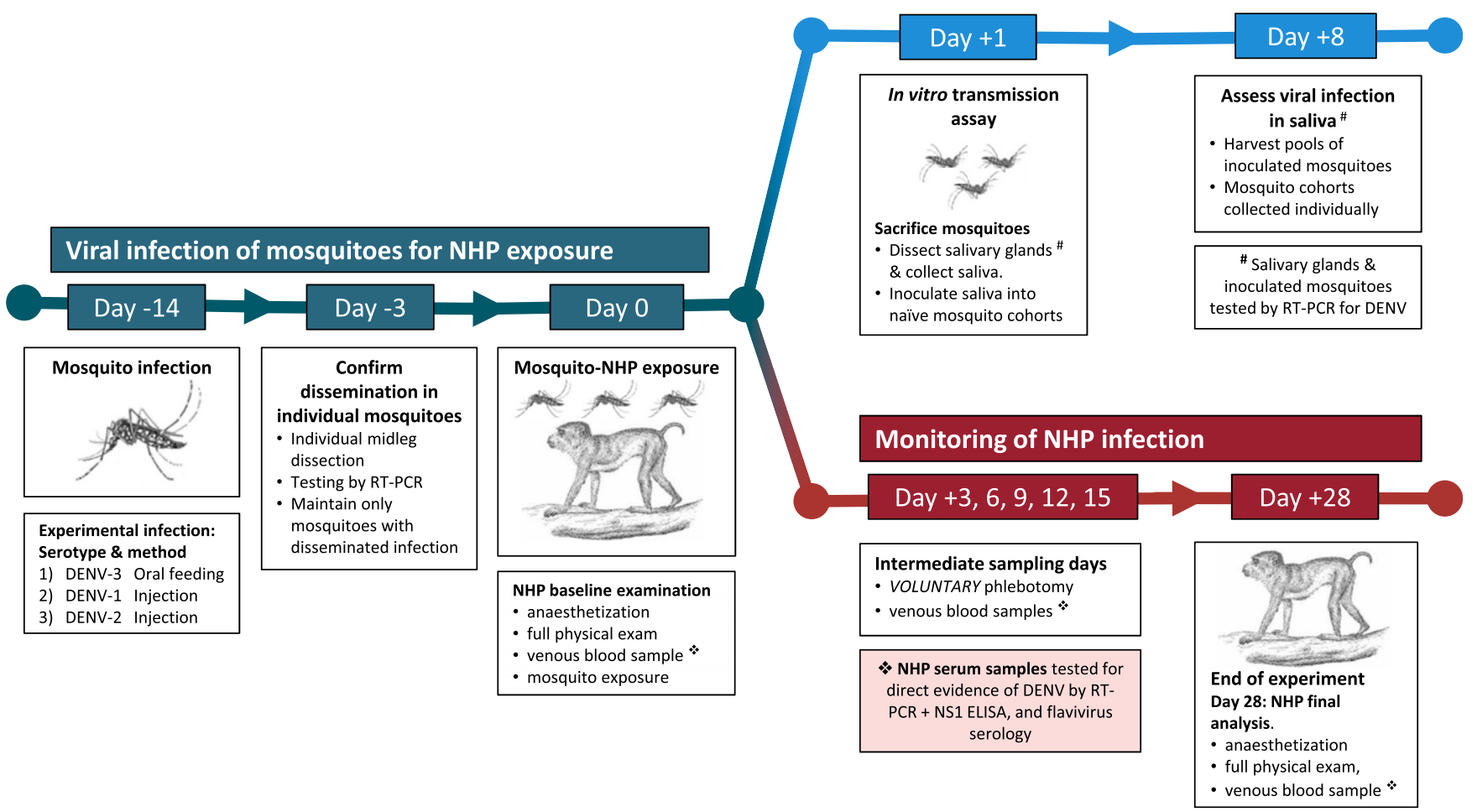

FIGURE 1. Overview of study design illustrating the infection of mosquitoes, followed by the independent assessments of in vitro transmission in mosquitoes and NHP infection over a 28-day time course. The schematic diagram illustrates the infection and sampling of mosquitoes and NHPs, sampling time points, and steps involved in each part of the study. Viral infection and incubation of mosquitoes took place from day 14-day 0 (in green, left hand side). The top branch of the diagram (in blue) shows the major steps involved in the IVT assay. The IVT assay was performed in parallel with the NHP infections, shown on the bottom branch of the figure (in red). Voluntary blood samples were collected from NHPs to test for evidence of dengue virus infection. Each experiment involved three NHPs, each fed upon by three mosquitoes, and the whole experiment was replicated three times (meaning a total of nine NHPs and 27 mosquitoes). IVT = in vitro transmission; NHP = nonhuman primate.

samples (days 0 and 28) were performed under anesthesia, in addition to a full physical examination. All veterinary care was administered by qualified AFRIMS veterinarians. The single human viremic blood sample used in this study was obtained via an ongoing study conducted by the AFRIMS (approval number WRAIR\#2093) granted by the Walter Reed Army Institute of Research Institutional Review Board.

Experimental preparation. Nonhuman primate preselection for experimentation. Approximately 6-12 months before experimentation, a number of individuals from the $M$. mulatta colony maintained at the AFRIMS were screened for potential inclusion in the experiment. Individuals of both sexes were prescreened to ensure they weighed more than $3.2 \mathrm{~kg}$ and were confirmed Flavivirus naive, using a hemagglutination inhibition assay (HIA). Naive individuals were recruited for subsequent training for voluntary phlebotomy (in accordance with the European Union regulations for working with NHPs), a process taking 4-6 months. A total of nine NHPs, all of which could be routinely phlebotomized safely, were finally enrolled in the study.

Mosquito rearing and origin. The mosquitoes in the first experiment were field-derived $\left(F_{0}\right)$, originating from the field in Nha Trang, central Vietnam. These mosquitoes were collected as eggs from ovitraps, as described in Carrington et al. ${ }^{10}$ Eggs were then delivered to the AFRIMS, Bangkok, for rearing and experimental use. In the latter two experiments, we used a field strain of Thai Ae. aegypti. These mosquitoes originated from
Kamphaeng Phet Province, collected from ovitraps in 2016, and colonized for one generation, before the $F_{1}$ generation was used in the experiments.

All mosquitoes used in the experiments were reared under standard laboratory conditions, at $26 \pm 1^{\circ} \mathrm{C}$, under a 12:12hour light: dark cycle. Larvae were reared at a density of approximately 300 larvae per $29 \times 39 \times 4.5-\mathrm{cm}$ plastic tray, filled with $2.5 \mathrm{~L}$ of dechlorinated water. They were fed daily with $0.8 \mathrm{~g}$ of fish food pellets (C.P. Hi Pro ${ }^{\circledR}$, Perfect Company Group Co. Ltd., Bangkok, Thailand).

Adult colonies (where appropriate) were maintained at the AFRIMS with human blood. Human blood purchased from the Thai Red Cross was used to feed Ae. aegypti in our mosquitorearing insectary. The Ae. aegypti colony was allowed to feed uninterrupted on the human blood for 1 hour using the artificial membrane technique and was maintained at $25 \pm 2^{\circ} \mathrm{C}$ and $80 \%$ relative humidity.

Viral infection of mosquitoes (day -14). On emergence as adults, a surplus of mosquitoes were maintained on $10 \%$ sucrose solution ad libitum until which time they were infected with DENV (either by oral feeding or inoculation).

In Experiment 1, mosquitoes were orally challenged with a patient-derived blood meal via artificial membrane feeders. The patient, suffering from a DENV-3 infection, was in their second day of illness at the time of enrollment. Plasma RNAemia was estimated at $3 \times 10^{8}$ genome copies $/ \mathrm{mL}$, according to quantitative Reverse Transcription (qRT)-PCR. 
Because of difficulties with patient recruitment after the first experiment, we were forced to infect mosquitoes parenterally in the remaining two experiments, using cell culture-grown virus. Dengue virus- 1 and DENV-2 were used in Experiments 2 and 3, respectively. Mosquitoes were inoculated with $\sim 1 \mu \mathrm{L}$ of DENV-1 (00442/05 B isolate, passaged 1 time in Toxorhynchites splendens mosquitoes, and five times in C6/36 cells) and DENV-2 (00210/15 isolate, passaged five times in C6/36 cells). The titer of the virus used for inoculation in both experiments was $4 \times 10^{6}$ plaque forming units (PFU)/mL (measured in rhesus monkey kidney cells).

After mosquitoes were exposed to virus, they were housed in paper cups (9-cm height $\times 8$-cm diameter) at a density of 15 females per cup and were maintained with $10 \%$ sucrose at $25 \pm 1^{\circ} \mathrm{C}$ for 14 days.

Testing for disseminated infection in virus-exposed mosquitoes (day -3). Virus-exposed mosquitoes were analyzed 3 days before being allowed to feed on NHPs, to determine whether the mosquitoes had a disseminated viral infection. A single midleg was dissected and placed in $150 \mu \mathrm{L}$ of RPMI medium supplemented with $10 \%$ fetal bovine serum. Leg samples were ground and homogenized using a bullet blender (Next Advance, New York, NY) at a speed of 8 for 3 minutes. Homogenized samples were tested by DENV qRT-PCR. After midleg dissection, mosquitoes were maintained individually in the new containers, and their path through the experiments could be traced with their unique identity. Only mosquitoes that tested PCR positive for DENV in leg samples were retained for possible feeding on the NHPs. Those mosquitoes with the highest titer leg infections were selected for the NHP-mosquito exposures, to be conducted on the study day 0 . An excess of mosquitoes were prepared to ensure any unengorged female could be replaced with a fresh mosquito as needed.

Infection of NHPs with direct mosquito bite (day 0). To enable mosquito feeding in a controlled manner, NHPs were anesthetized with ketamine by a registered veterinary doctor. Eight milliliters of venous blood was collected before mosquito exposure to reestablish a negative baseline Flavivirus serology.

For each experiment, three anesthetized NHPs were each exposed to three mosquitoes with a PCR-confirmed disseminated DENV infection. The mosquitoes chosen to use in the exposures were those with the highest viral concentration in the leg tissues. Mosquitoes were allowed 15 minutes to feed to repletion on the skin of the NHP; if they did not feed at that time, they were replaced with the mosquitoes with the next highest leg titer. We closely monitored and observed mosquito's probing and feeding activity at all times; when mosquitoes were inactive and did not probe/feed on an NHP, they were replaced. A maximum of three mosquitoes fed to repletion on each NHP, and after feeding, mosquitoes were retained for subsequent in vitro testing (see next section).

In vitro virus transmission by mosquitoes (days 1 and 8). We performed the standard IVT assay using the same mosquitoes that had fed upon NHPs the day prior. In performing the IVT assay 1 day later (day 1), we aimed at ensuring mosquitoes had replenished their saliva.

Mosquitoes were killed in the conventional laboratorybased IVT assay, with saliva collected from de-legged/ de-winged mosquitoes, before subsequent salivary gland dissection. Salivary glands were tested directly by RT-PCR to determine the presence of viral RNA. Each saliva sample was inoculated into 5-6 virus-naive Ae. aegypti mosquitoes, which were then incubated for 7 days (day 8), allowing for any virus present in the saliva to amplify within the inoculated mosquitoes. At the completion of the incubation period, the inoculated Ae. aegypti from a single index mosquito were pooled and tested for the presence of virus by PCR (see Diagnostics section). A positive result was interpreted, as a mosquito that had transmission potential in the IVT assay. The unique identity and infection status of each individual were then associated with the infection status of the NHP on which it fed.

Routine monitoring of NHPs for infection (day 3 to 28). After the mosquito-NHP exposure on day 0 , NHPs were monitored with voluntary blood draws of 1-2 $\mathrm{mL}$ volume, every 3 days until day 15. Blood was collected from the cephalic vein in the arm. As all blood draws were voluntary, if an NHP was refused to provide a blood sample on a given day, a second attempt would be made on that day; if the second attempt to draw blood was refused, that blood sample would be forsaken. The final blood draw, taken on day 28 , was performed while the NHP was under anesthesia, along with a routine physical examination to ensure the NHP remained in good health. As with the first blood sample, this final $8 \mathrm{~mL}$ was collected from the saphenous vein in the leg. Serum was separated from each blood sample and prepared into three aliquots, assigned for each screening assay. We tested for 1) DENV NS1 antigen, 2) IgM and IgG antibody responses to both DENV and JEV antigens, and 3) DENV RNA in a nested RT-PCR.

Diagnostic testing of samples. Mosquito sample processing. At respective time points, mosquito tissues (legs, salivary glands, or pooled inoculated mosquito samples) were homogenized in RPMI 1640 (Sigma Aldrich, St. Louis, MO) medium mixed with $10 \%$ FBS. A $140 \mu \mathrm{L}$ sample of the homogenate was used for nucleic acid extraction using the QIAamp Viral RNA Mini Kit (Qiagen, Hilden, Germany). All mosquito tissues were screened by RT-PCR for DENV infection as described in the Molecular Diagnostics section. Measurements for mosquito samples were returned in genome equivalents (GE)/150 $\mu \mathrm{L}$ of the homogenate for mosquitoes.

Nonhuman primates sample processing. A serum sample was collected from each NHP for HIA testing between 10 and 52 days before experimental exposure to mosquitoes. Samples were tested by HIA using the method described in Clarke and Casels. ${ }^{18}$ Samples with a titer $<10$ were considered negative for past Flavivirus infection.

Postexposure to mosquitoes, sera from the NHPs were tested for NS1 antigen in the blood using BioRad's NS1 ELISA kit, following the manufacturer's instructions. Results are presented in the sample-to-cutoff ratio, where values greater than 1 were considered positive and those less than 0.5 were considered negative. Values between 0.5 and 1 were considered equivocal; the first time an equivocal result was observed, the test was performed a second time. The second result was used as the final result. Antibody responses were investigated using an enzyme immune assay (EIA), as per Kato et al. $^{19}$ The $\operatorname{lgM}$ and $\operatorname{lgG}$ results were considered positive if there was a 4-fold rise in EIA units between days 0 and either day 14 or 28 . The same cutoff was used for both DENV and JEV assays. Sera samples were also tested for the presence of viral RNA after RNA extractions using the QIAamp Viral RNA Mini Kit, as per the manufacturer's instruction. Viral RNA was detected using the TaqMan real-time RT-PCR method as 
described by Klungthong et al. ${ }^{20}$ Samples were considered positive if the cycle threshold values were less than 40 . Viral titers were calculated for each sample and reported in GEs/mL of serum.

Data analysis. As the aim of this work was to explore the reliability and features of DENV infection delivered by direct mosquito bites to NHPs, we provide a descriptive summary of the kinetics of RNAemia, NS1 antigenemia, and $\operatorname{lgM} / \mathrm{lgG}$ antibodies. We also describe the prevalence of virus transmission using the IVT assay relative to the NHP infection model. All graphical outputs were produced using $\mathrm{R}$ software (RStudio Team 2015, Boston, MA).

\section{RESULTS}

We performed three DENV infection experiments, each with three NHPs fed upon by three mosquitoes (a total of nine rhesus monkeys and 27 mosquitoes). On each of the scheduled sampling days, all NHPs volunteered blood samples, meaning the sampling regime (Figure 1) was complete for analysis. In the first experiment, NHPs fed upon by mosquitoes that had been orally infected with DENV-3 did not result in infection (negative for NS1, RT-PCR for viral RNA, and lgM/lgG ELISA).

The subsequent two experiments, each using mosquitoes that had been intrathoracically inoculated with DENV-1 and DENV-2, both resulted in a 100\% attack rate. Descriptive results of each experiment for both the mosquito and the associated NHPs, are detailed in the following texts, alongside a summary of the results for all three experiments presented in Table 1.

Experiment 1: Mosquito infection via oral challenge with patient-derived DENV-3 blood meal. Mosquitoes in Experiment 1 were orally infected with a patient-derived DENV-3 viremic blood meal (>10 8 viral genome copies $/ \mathrm{mL}$ ). A total of 96.5\% (28/29) of mosquitoes developed a disseminated leg infection in pre-exposure screening. The final cohort of nine mosquitoes that fed upon the NHPs had DENV-positive salivary glands, with a minimum viral load of $4.9 \times 10^{3} \mathrm{GE}$ copies of virus/sample (Figure 2). Most of these nine mosquitoes also had saliva that tested positive for DENV (Table 1). However, we failed to detect evidence of DENV infection in any of the NHPs (Table 1, Figures 3 and 4).

Experiment 2: Mosquito infection via intrathoracic inoculation of cell-cultured DENV-1. In Experiment 2, mosquitoes were injected with $\sim 1 \mu \mathrm{L}$ of $4 \times 10^{6}$ genome copies $/ \mathrm{mL}$ DENV-1 serotype. All 50 index mosquitoes inoculated with cultured virus developed disseminated leg infections. The lowest viral load among the dissected salivary glands from these mosquitoes was $4 \times 10^{4} \mathrm{GE}$ virus/homogenate. Eight of nine mosquitoes also had saliva that tested positive for DENV (Table 1). All three NHPs had virological and serological evidence of DENV infection after being fed upon. Two individuals (R1112 and R1141) had detectable RNAemia on days 3, 6 , and 9 , with peak titers observed on day $6\left(\sim 10^{5} \mathrm{GE}\right.$ virus $/ 1 \mathrm{~mL}$

TABLE 1

Summary of test results for mosquitoes and NHPs after infection with DENV-1, 2, or 3

\begin{tabular}{|c|c|c|c|c|c|c|c|c|c|c|}
\hline \multirow[b]{2}{*}{$\begin{array}{l}\text { Summary of experimental } \\
\text { details }\end{array}$} & \multicolumn{5}{|c|}{ Mosquito results (from RT-PCR) } & \multicolumn{5}{|c|}{ NHP results (qualitative summary across all time points) } \\
\hline & $\begin{array}{l}\text { Mosquito } \\
\text { replicate }\end{array}$ & $\begin{array}{l}\text { Index } \\
\text { mosquito } \\
\text { number* }\end{array}$ & $\begin{array}{l}\text { Dissected } \\
\text { legs }\end{array}$ & $\begin{array}{l}\text { Dissected } \\
\text { salivary } \\
\text { glands }\end{array}$ & $\begin{array}{c}\text { Pool of } \\
\text { inoculated } \\
\text { mosquitoes }\end{array}$ & NHP ID & $\begin{array}{l}\text { Viremia } \\
\text { (RT-PCR) }\end{array}$ & $\begin{array}{l}\text { NS1 antigenemia } \\
\text { (enzyme immune } \\
\text { assay) }\end{array}$ & $\begin{array}{l}\text { DENV IgM } \\
\text { (ELISA) }\end{array}$ & $\begin{array}{l}\text { DENV IgG } \\
\text { (ELISA) }\end{array}$ \\
\hline \multirow{3}{*}{$\begin{array}{l}\text { Experiment 1: Patient- } \\
\text { derived blood meal } \\
\text { administered by oral } \\
\text { challenge for } F_{0} \\
\text { mosquitoes from } \\
\text { Vietnam }\end{array}$} & $\begin{array}{l}1 \\
2 \\
3\end{array}$ & $\begin{array}{l}14 \\
20 \\
27\end{array}$ & $\begin{array}{l}\text { Positive } \\
\text { Positive } \\
\text { Positive }\end{array}$ & $\begin{array}{l}\text { Positive } \\
\text { Positive } \\
\text { Positive }\end{array}$ & $\begin{array}{l}\text { Positive } \\
\text { Negative } \\
\text { Positive }\end{array}$ & R762 & Negative & Negative & Negative & Negative \\
\hline & $\begin{array}{l}4 \\
5 \\
6\end{array}$ & $\begin{array}{c}\text { Gr3.1 } \\
\text { Gr3.2 } \\
21\end{array}$ & $\begin{array}{l}\text { Positive } \\
\text { Positive } \\
\text { Positive }\end{array}$ & $\begin{array}{l}\text { Positive } \\
\text { Positive } \\
\text { Positive }\end{array}$ & $\begin{array}{l}\text { Negative } \\
\text { N/A } \\
\text { N/A }\end{array}$ & R1014 & Negative & Negative & Negative & Negative \\
\hline & $\begin{array}{l}7 \\
8 \\
9\end{array}$ & $\begin{array}{c}\text { Gr2.1 } \\
10 \\
19\end{array}$ & $\begin{array}{l}\text { Positive } \\
\text { Positive } \\
\text { Positive }\end{array}$ & $\begin{array}{l}\text { Positive } \\
\text { Positive } \\
\text { Positive }\end{array}$ & $\begin{array}{l}\text { Positive } \\
\text { Negative } \\
\text { Positive }\end{array}$ & R1054 & Negative & Negative & Negative & Negative \\
\hline \multirow{3}{*}{$\begin{array}{l}\text { Experiment 2: } \\
\text { DENV-1 cell } \\
\text { culture-grown virus } \\
\text { inoculated into the } \\
\text { thorax of } F_{1} \\
\text { mosquitoes from } \\
\text { Thailand }\end{array}$} & $\begin{array}{l}10 \\
11 \\
12\end{array}$ & $\begin{array}{r}6 \\
7 \\
20\end{array}$ & $\begin{array}{l}\text { Positive } \\
\text { Positive } \\
\text { Positive }\end{array}$ & $\begin{array}{l}\text { Positive } \\
\text { Positive } \\
\text { Positive }\end{array}$ & $\begin{array}{l}\text { Positive } \\
\text { Positive } \\
\text { Positive }\end{array}$ & R1112 & Positive & Positive & Positive & Positive \\
\hline & $\begin{array}{l}13 \\
14 \\
15\end{array}$ & $\begin{array}{r}1 \\
14 \\
16\end{array}$ & $\begin{array}{l}\text { Positive } \\
\text { Positive } \\
\text { Positive }\end{array}$ & $\begin{array}{l}\text { Positive } \\
\text { Positive } \\
\text { Positive }\end{array}$ & $\begin{array}{l}\text { Positive } \\
\text { Positive } \\
\text { Positive }\end{array}$ & R1138 & Positive & Positive & Positive & Positive \\
\hline & $\begin{array}{l}16 \\
17 \\
18\end{array}$ & $\begin{array}{l}45 \\
40 \\
39\end{array}$ & $\begin{array}{l}\text { Positive } \\
\text { Positive } \\
\text { Positive }\end{array}$ & $\begin{array}{l}\text { Positive } \\
\text { Positive } \\
\text { Positive }\end{array}$ & $\begin{array}{l}\text { Positive } \\
\text { Negative } \\
\text { Positive }\end{array}$ & R1141 & Positive & Positive & Positive & Positive \\
\hline \multirow{3}{*}{$\begin{array}{l}\text { Experiment 3: } \\
\text { DENV-2 cell } \\
\text { culture-grown virus } \\
\text { inoculated into the } \\
\text { thorax of } F_{1} \\
\text { mosquitoes from } \\
\text { Thailand }\end{array}$} & $\begin{array}{l}19 \\
20 \\
21\end{array}$ & $\begin{array}{l}26 \\
42 \\
48\end{array}$ & $\begin{array}{l}\text { Positive } \\
\text { Positive } \\
\text { Positive }\end{array}$ & $\begin{array}{l}\text { Positive } \\
\text { Positive } \\
\text { Positive }\end{array}$ & $\begin{array}{l}\text { Positive } \\
\text { Positive } \\
\text { Positive }\end{array}$ & R710 & Positive & Positive & Positive & Positive \\
\hline & $\begin{array}{l}22 \\
23 \\
24\end{array}$ & $\begin{array}{l}22 \\
35 \\
36\end{array}$ & $\begin{array}{l}\text { Positive } \\
\text { Positive } \\
\text { Positive }\end{array}$ & $\begin{array}{l}\text { Positive } \\
\text { Positive } \\
\text { Positive }\end{array}$ & $\begin{array}{l}\text { Positive } \\
\text { Positive } \\
\text { Positive }\end{array}$ & R1007 & Negative & Positive & Positive & Positive \\
\hline & $\begin{array}{l}25 \\
26 \\
27\end{array}$ & $\begin{array}{l}44 \\
58 \\
61\end{array}$ & $\begin{array}{l}\text { Positive } \\
\text { Positive } \\
\text { Positive }\end{array}$ & $\begin{array}{l}\text { Positive } \\
\text { Positive } \\
\text { Positive }\end{array}$ & $\begin{array}{l}\text { Positive } \\
\text { Positive } \\
\text { Positive }\end{array}$ & R1146 & Negative & Positive & Positive & Positive \\
\hline
\end{tabular}

DENV = dengue virus; NHP = nonhuman primate; $\mathbf{q}=$ no mosquitoes from these cohorts survived to the day of harvesting. Mosquito tissue results represent the qualitative test result. NHP results represent the qualitative result from respective tests, with at least one "positive" test result across any time point for a particular test being considered as a positive result for that assay overall.

* Mosquitoes listed with "GrX.X" were initially grouped together in a single cup for the actual mosquito-NHP exposure. Therefore, their individual leg titers could not be linked to their eventual transmission potential because their unique identity was unlinked when they were grouped. All other mosquitoes can be traced from the beginning to the end. 


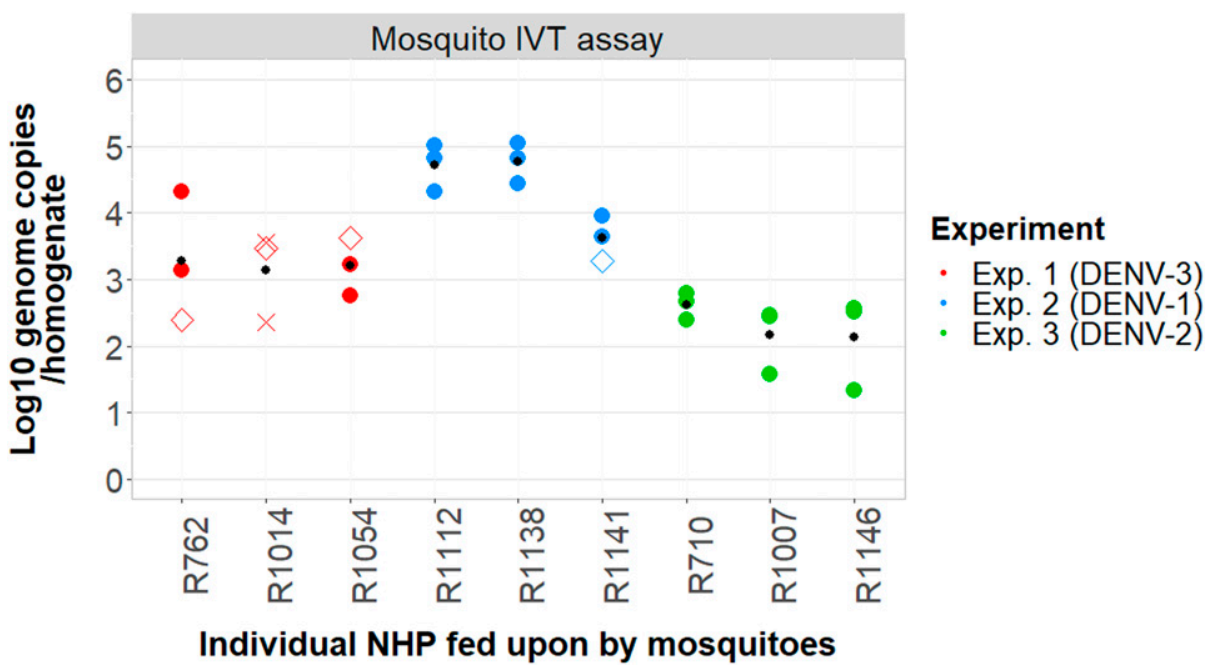

FIGURE 2. Infection results for mosquitoes tested in the in vitro transmission (IVT) assay, indicating the viral load in the salivary glands and the transmission status of each mosquito. Each data point represents the $\log _{10}$ viral load of the salivary glands from a single mosquito, as measured by RT-PCR. These points are plotted against the nonhuman primate (NHP) on which they fed (3 mosquitoes for each NHP). Mosquitoes were tested for IVT 1 day after they had fed upon the NHPs. Each data point represents a single mosquito that was exposed to virus and fed upon one of the NHPs. Closed colored circles $(\bullet)$ represent mosquitoes with evidence of virus in their saliva in the IVT assay, open colored diamonds $(\diamond)$ represent mosquitoes with no evidence of virus transmission, crosses $(X)$ represent mosquitoes for which no data are available about their transmission status (i.e., all the mosquitoes that were inoculated with their saliva died before sampling). The smaller black dot (•) represents the average viral titer in the salivary glands of those three mosquitoes that fed upon each respective NHP.

serum). Detection of RNAemia in NHP R1138 was observed on days 6 and 9 only and peaked $\sim 2$ logs lower than the other two NHPs (Figure 3). Nonhuman primate R1112, which had the highest RNAemia overall, had detectable NS1 circulating on days $6,9,12$, and 15 . Nonhuman primate R1141 was NS1 antigenemic on days 9 and 12 only. Nonhuman primate
R1138, which had the delayed onset of RNAemia, was also delayed in the detection of DENV NS1 (Figure 3). In all individuals, DENV-reactive lgM was the highest at the day 15 time point, with > 50 EIA units (but may have peaked either before or after), but remained detectable at day 28. Dengue virusreactive IgG was detected on either day 15 or 28 (Figure 4).
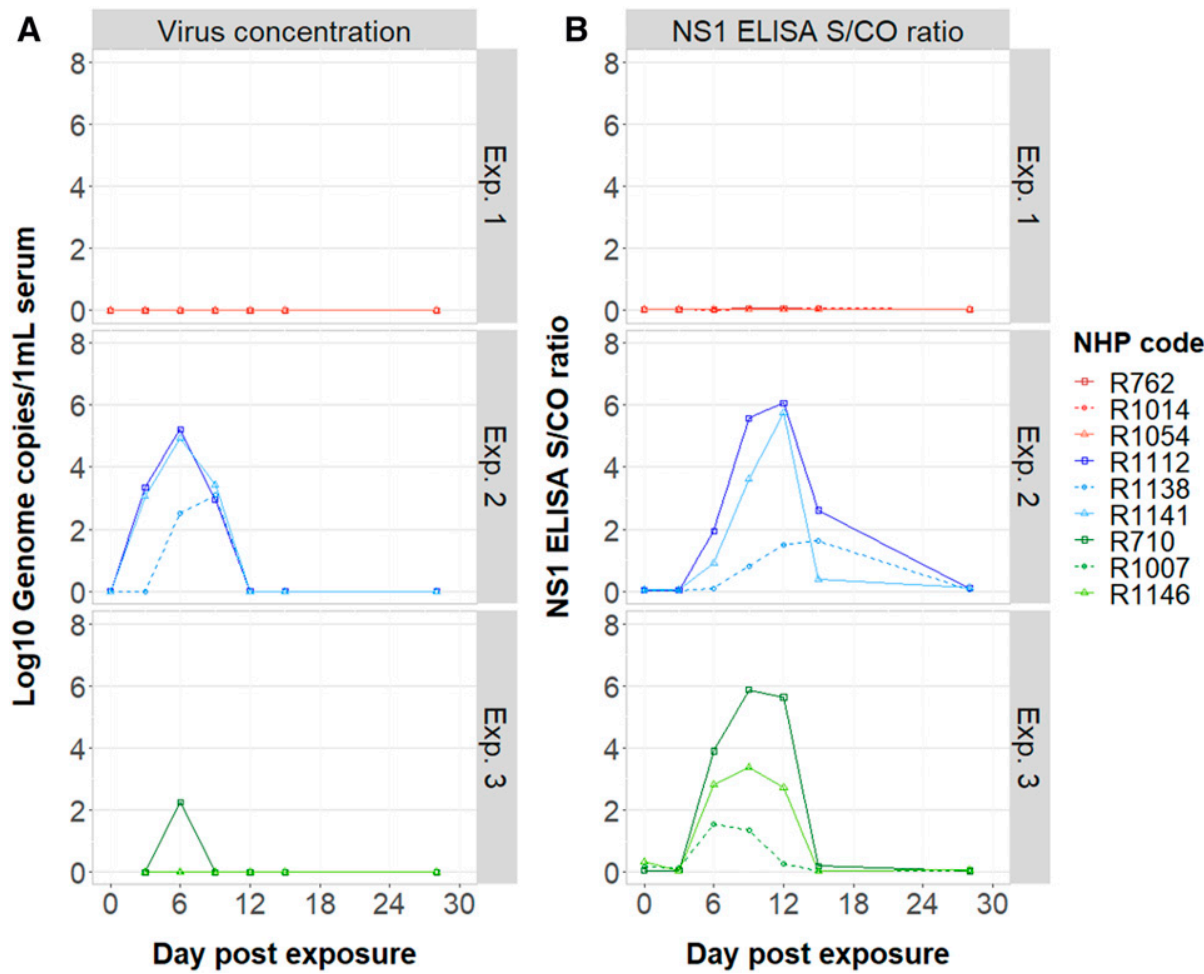

FIGURE 3. Temporal virological responses observed in nonhuman primates (NHPs) exposed to dengue virus (DENV) via direct mosquito bites. Data are stratified by experiment. (A) Changes in viral concentration in the serum, as measured by RT-PCR. (B) Detection of DENV NS1 antigenemia (measured by enzyme immune assay). The signal-to-cutoff $(\mathrm{S} / \mathrm{CO})$ ratio of the assay is plotted as a function of the day at which the NHPs were sampled. 


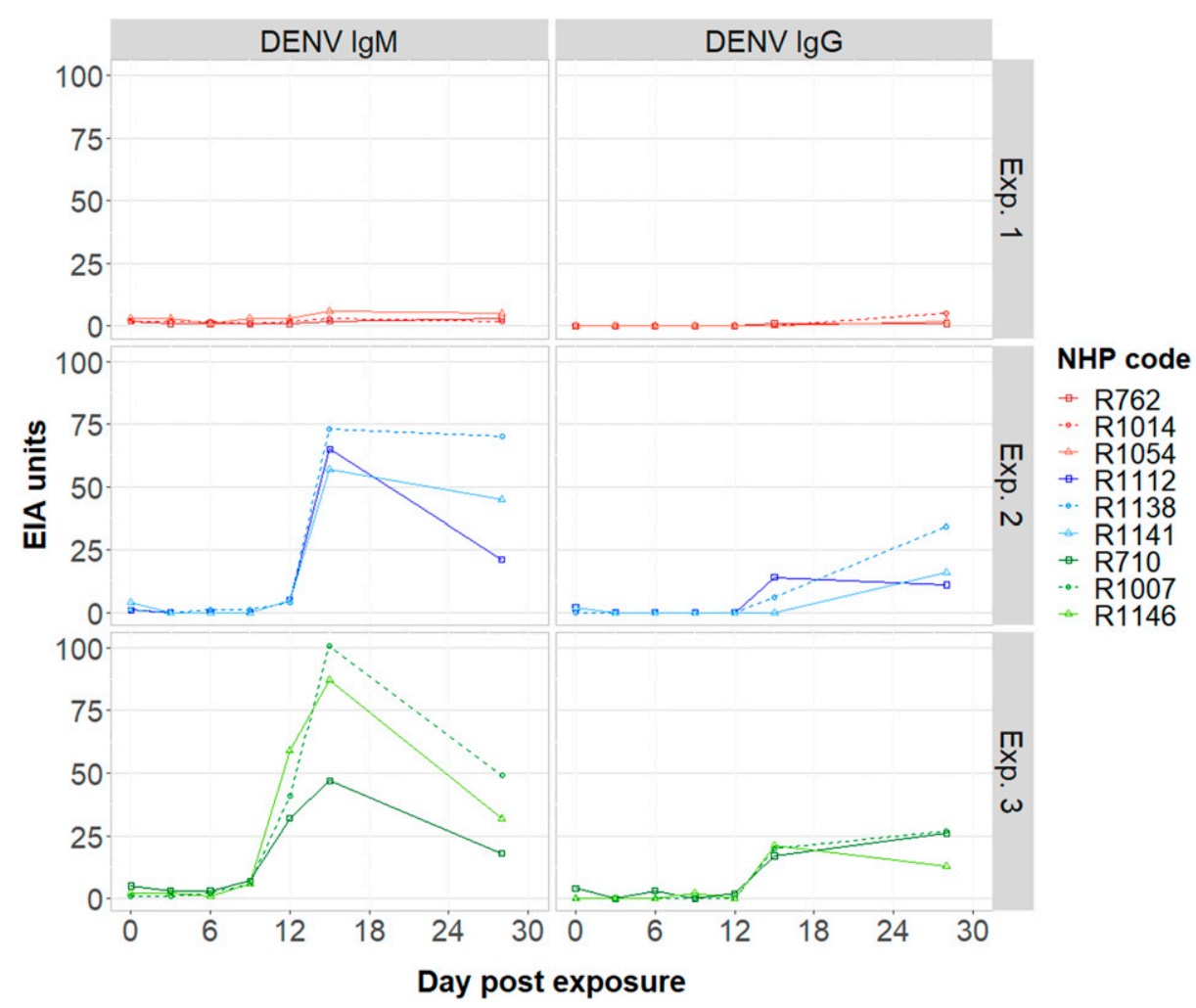

FIGURE 4. Temporal immunological responses of nonhuman primates (NHPs) after infection with dengue virus (DENV), stratified by experiment. The figure shows the serological changes, measured by ELISA, for DENV IgM/lgG according to the day of NHP sampling.

Experiment 3: Mosquito infection via intrathoracic inoculation of cell-cultured DENV-2. In Experiment 3, mosquitoes were again inoculated with virus but this time with DENV-2. Consistent with the previous injection experiment, $100 \%$ of inoculated mosquitoes developed disseminated leg and salivary gland infections. Viral load in the mosquito salivary glands ranged between $4.5 \times 10^{2}$ and $1.3 \times 10^{4} \mathrm{GE}$ virus/ homogenate. Despite the average salivary gland virus titer of these nine engorged mosquitoes being lower than that of the nine engorged mosquitoes in Experiment 1 (Figure 2), all females in this experiment tested positive for virus in their saliva in the IVT assay. All three NHPs developed DENV infection. NS1 was positive on days 6,9 , and 12 in all individuals (Figure 3), and although IgM became detectable on day 12, its peak was on day 15. The highest IgM measurements were observed in the single individual that had a detectable RNAemia (R710; $1.7 \times 10^{2}$ GE virus/1 mL serum; Figure 4). IgG was detected on days 15 and 28 in all three individuals.

\section{DISCUSSION}

One of the aims of this study was to characterize the NHP response to virus infection by direct mosquito bites, after feeding on a human patient-derived blood meal. Although unsuccessful in this particular goal, we were able to infect six NHPs following the direct bites of mosquitoes parenterally infected with virus, and characterize the viral kinetics and immune response profiles in these NHPs. In doing so, we report our descriptive comparison of the IVT and NHP infection.

In total, $100 \%$ of NHPs were DENV-infected after receiving bites of Ae. aegypti mosquitoes that had been infected by intrathoracic inoculation. RNAemia was detected in all three NHPs exposed to DENV-1 and one NHP exposed to DENV-2. Interestingly, the two individuals without DENV-2 RNAemia had the highest concentrations of anti-DENV antibodies. Although our 3-day intervals between sampling may have led us to miss a small window in which RNAemia may have been detectable, it is plausible that in these animals, a robust antibody response substantially reduced the magnitude and duration of the viremia. ${ }^{21}$ As also observed in human patients infected with DENV-2, ${ }^{22}$ we noted individual NHPs with low NS1 also had lower (or non-detectable) levels of RNAemia.

Overall, the onset and duration of RNAemia among NHPs infected by direct mosquito bites are similar to those subcutaneously inoculated with DENV. ${ }^{11,23}$ Likewise, Hickey et al. ${ }^{15}$ demonstrated that after inoculating rhesus monkeys with $10^{5} \mathrm{PFU} / \mathrm{mL}$ of each of four DENV serotypes, IgM titers were at maximal levels (based on the time points tested) on either day 10 or 14. In our own experiments, a quick increase in IgM levels and peak were observed, in response to DENV-1 and DENV-2 infections, around day 15.

Surprisingly, when we used Ae. aegypti that had been infected with DENV-3 by oral feeding on the dengue patient's blood, we failed to detect any evidence of infection in the three NHPs. This was despite all nine index mosquitoes having DENV-infected salivary glands and four of seven mosquitoes possessing infectious virus in their saliva. Pinpointing contributing factor(s) to explain this outcome is challenging, however, given a number of factors were changed between experiments, including the use of clinical versus cell-cultured virus isolates, mosquito infection via oral feeding versus intrathoracic inoculation, different virus serotypes and virus 
titers, and different origins of the mosquito populations. Plausibly, these three NHPs may have been inherently resistant to DENV infection (although serologically they were Flavivirus naive). Subsequent DENV challenge of the NHPs in question by inoculation with cultured virus could help test this hypothesis. DENV-3 is able to infect NHPs ${ }^{23}$; however, the particular DENV-3 in question might have been inherently less infectious than the DENV-1 and DENV-2 we used. If we had used more animals per group or used more infected mosquitoes per animal, we might have observed a different outcome.

A limitation of this pilot study is that it is unknown whether a single mosquito or multiple mosquitoes are responsible for delivering the infectious bite(s) to the naive NHPs. Given our inability to reliably infect NHPs using DENV-infected mosquitoes, further work is still needed to develop an epidemiologically relevant human-mosquito-NHP infection model capable of testing the efficacy of novel mosquito interventions such as Wolbachia. Despite this, our work identifies reliable time points for screening DENV infection in this species after being fed upon by DENV-1- and DENV-2-infected mosquitoes to maximize the detection of viremia, NS1 antigenemia, and DENV-reactive antibodies.

Received August 26, 2019. Accepted for publication March 25, 2020. Published online May 18, 2020.

Acknowledgments: We would like thank the World Mosquito Program Nha Trang field team for the collection and delivery of mosquitoes and Dr. Anon Srikiatkhachorn for the provision of viremic patients' blood samples.

Financial support: This work was funded by a Wellcome Trust grant to CPS.

Disclosure: All authors reviewed and approved the manuscript before submission.

Disclaimer: The material has been reviewed by the Walter Reed Army Institute of Research. There is no objection to its presentation and/or publication. The opinions or assertions contained herein are the private views of the author, and are not to be construed as official, or as reflecting true views of the Department of the Army or the Department of Defense. Research was conducted under an approved animal use protocol in an AAALACi-accredited facility in compliance with the Animal Welfare Act and other federal statutes and regulations relating to animals and experiments involving animals and adheres to the principles stated in the Guide for the Care and Use of Laboratory Animals, NRC Publication, 2011 edition. The investigators have adhered to the policies for the protection of human subjects as prescribed in AR 70-25.

Authors' addresses: Lauren B. Carrington, Oxford University Clinical Research Unit (OUCRU), Wellcome Trust Asia-Africa Programme, Ho Chi Minh City, Vietnam, and Nuffield Department of Medicine, University of Oxford, Oxford, United Kingdom, E-mail: Ibcarrington@ gmail.com. Alongkot Ponlawat, Chanyapat Nitatsukprasert, and Patcharee Khongtak, Entomology Department, Armed Forces Research Institute of Medical Sciences (AFRIMS), Bangkok, Thailand, E-mails: alongkotp.fsn@afrims.org, chanyapatn.fsn@afrims.org, and patchareek.fsn@afrims.org. Piyanate Sunyakumthorn, Christine A. Ege, Rawiwan Im-erbsin, and Kesara Chumpolkulwong, Veterinary Medicine Department, Armed Forces Research Institute of Medical Sciences (AFRIMS), Bangkok, Thailand, E-mails: piyanates.fsn@ afrims.org, christine.ege.mil@afrims.org, rawiwani.fsn@afrims.org, and kesarac.fsn@afrims.org. Butsaya Thaisomboonsuk, Chonticha Klungthong, and Louis Macareo, Virology Department, Armed Forces Research Institute of Medical Sciences (AFRIMS), Bangkok, Thailand, E-mails: butsayat.fsn@afrims.org, chontichak.fsn@afrims.org, and louis.macareo.mil@afrims.org. In-Kyu Yoon, Virology Department, Armed Forces Research Institute of Medical Sciences (AFRIMS), Bangkok, Thailand, and Global Dengue and Aedes-transmitted Diseases Consortium, Seoul, South Korea, E-mail: inkyu.yoon@ivi.int.
Damon Ellison, Virology Department, Armed Forces Research Institute of Medical Sciences (AFRIMS), Bangkok, Thailand, and Walter Reed Army Institute of Research (WRAIR), Silver Spring, MD, E-mail: damon.w.ellison.mil@mail.mil. Cameron P. Simmons, Oxford University Clinical Research Unit (OUCRU), Wellcome Trust Asia-Africa Programme, Ho Chi Minh City, Vietnam, Nuffield Department of Medicine, University of Oxford, Oxford, United Kingdom, and Institute for Vector-Borne Diseases, Monash University, Melbourne, Australia, E-mail: cameron.simmons@worldmosquito.org.

This is an open-access article distributed under the terms of the Creative Commons Attribution (CC-BY) License, which permits unrestricted use, distribution, and reproduction in any medium, provided the original author and source are credited.

\section{REFERENCES}

1. Ferguson NM et al., 2015. Modeling the impact on virus transmission of Wolbachia-mediated blocking of dengue virus infection of Aedes aegypti. Sci Transl Med 7: 279ra37.

2. Nazni WA et al., 2019. Establishment of Wolbachia strain wAlbB in Malaysian populations of Aedes aegypti for dengue control. Curr Biol 29: 4241-4248.e5.

3. Ryan PA et al., 2019. Establishment of wMel Wolbachia in Aedes aegypti mosquitoes and reduction of local dengue transmission in Cairns and surrounding locations in northern Queensland, Australia. Gates Open Res 3: 1547.

4. Aitken THG, 1977. An in vitro feeding technique for artificially demonstrating virus transmission by mosquitoes. Mosquito News 37: 130-133.

5. Nguyen NM et al., 2013. Host and viral features of human dengue cases shape the population of infected and infectious Aedes aegypti mosquitoes. Proc Natl Acad Sci USA 110: 9072-9077.

6. Whitehorn $\mathrm{J}$ et al., 2015. Comparative susceptibility of Aedes albopictus and Aedes aegypti to dengue virus infection after feeding on blood of viremic humans: implications for public health. J Infect Dis 212: 1182-1190.

7. Danforth ME, Reisen WK, Barker CM, 2016. The impact of cycling temperature on the transmission of west nile virus. $J$ Med Entomol 53: 681-686.

8. Dubrulle M, Mousson L, Moutailler S, Vazeille M, Failloux AB, 2009. Chikungunya virus and Aedes mosquitoes: saliva is infectious as soon as two days after oral infection. PLoS One 4: 6.

9. Kilpatrick AM, Meola MA, Moudy RM, Kramer LD, 2008. Temperature, viral genetics, and the transmission of West Nile virus by Culex pipiens mosquitoes. PLoS Pathog 4: 7.

10. Carrington LB et al., 2018. Field- and clinically derived estimates of Wolbachia-mediated blocking of disseminated dengue virus infection in Aedes aegypti mosquitoes. Proc Natl Acad Sci USA 115: 361-366.

11. Halstead SB, Shotwell H, Casals J, 1973. Studies on pathogenesis of dengue infection in monkeys. 1. Clinical laboratory responses to primary infection. J Infect Dis 128: 7-14.

12. Halstead SB, Shotwell H, Casals J, 1973. Studies on pathogenesis of dengue infection in monkeys. 2. Clinical laboratory responses to heterologous infection. J Infect Dis 128: 15-22.

13. Halstead SB, Casals J, Shotwell H, Palumbo N, 1973. Studies on immunization of monkets against dengue 1. Protection derived from single and sequential virus-infections Am J Trop Med Hyg 22: 365-374.

14. Halstead SB, Palumbo NE, 1973. Studies on immunization of monkeys against dengue. 2. Protection following inoculation of combinations of viruses. Am J Trop Med Hyg 22: 375-381.

15. Hickey AC, Koster JA, Thalmann CM, Hardcastle K, Tio PH, Cardosa MJ, Bossart KN, 2013. Serotype-specific host responses in rhesus macaques after primary dengue challenge. Am J Trop Med Hyg 89: 1043-1057.

16. Watts DM, Burke DS, Harrison BA, Whitmire RE, Nisalak A, 1987. Effect of Temperature on the vector efficiency of Aedes aegypti for Dengue 2 virus. Am J Trop Med Hyg 36: 143-152.

17. Dudley DM et al., 2017. Infection via mosquito bite alters Zika virus tissue tropism and replication kinetics in rhesus macaques. Nat Commun 8: 2096. 
18. Clarke $\mathrm{DH}$, Casals J, 1958. Techniques for hemagglutination and hemagglutination-inhibition with arthropod-borne viruses. Am J Trop Med Hyg 7: 561-573.

19. Kato F, Ishida Y, Kawakami A, Takasaki T, Saijo M, Miura T, Hishiki $T, 2018$. Evaluation of Macaca radiata as a non-human primate model of dengue virus infection. Scientific Rep 8.

20. Klungthong $C$ et al., 2015. Monitoring and improving the sensitivity of dengue nested RT-PCR used in longitudinal surveillance in Thailand. $J$ Clin Virol 63: 25-31.

21. Clapham H, Than HQ, Duong THK, Dorigatti I, Simmons CP, Ferguson NM, 2016. Modelling virus and antibody dynamics during dengue virus infection suggests a role for antibody in virus clearance. PLoS Comput Biol 12: e1004951.

22. Libraty DH, Young PR, Pickering D, Endy TP, Kalayanarooj S, Green S, Vaughn DW, Nisalak A, Ennis FA, Rothman AL, 2002. High circulating levels of the dengue virus nonstructural protein NS1 early in dengue illness correlate with the developement of dengue hemorrhagic fever. $J$ Infect Dis 186: $1165-1168$.

23. Althouse BM, Durbin AP, Hanley KA, Halstead SB, Weaver SC, Cummings DA, 2014. Viral kinetics of primary dengue virus in non-human primates: a systematic review and individual pooled analaysis. Virology 452-453: 237-246. 